\title{
Genome-wide DNA methylation analysis in permanent atrial fibrillation
}

\author{
GUOCHANG ZHAO, JIAN ZHOU, JIE GAO, YAN LIU, SONG GU, XITAO ZHANG and PIXIONG SU \\ Department of Cardiac Surgery, Beijing Chaoyang Hospital, \\ Capital Medical University, Ministry of Education, Beijing 100020, P.R. China
}

Received June 7, 2016; Accepted April 25, 2017

DOI: $10.3892 / \mathrm{mmr} .2017 .7221$

\begin{abstract}
Atrial fibrillation (AF) is a highly heterogeneous genetic disease; however, the pathogenesis of AF cannot be explained by genetic variants alone. DNA methylation is a heritable method of gene expression regulation, and may be a potential regulatory mechanism in AF. Therefore, in the present study, the genome-wide DNA methylation pattern in cells derived from the left atrium of patients with permanent AF $(n=7)$ was compared with that of healthy heart donors $(n=4)$ with a normal sinus rhythm (SR). Enriched biological functions of the differentially methylated genes were assessed. Integrated analysis of genome-wide methylation and mRNA expression profiles was performed, and reverse transcription quantitative-polymerase chain reaction (RT-qPCR) was used to determine the expression levels of four selected genes. A total of 417 differentially methylated $\mathrm{CpG}$ sites were identified in the fibrillating atrium $(\mathrm{P}<0.05 ;|\beta|>0.17)$; the majority of which were located in gene-body and intergenic regions outside of $\mathrm{CpG}$ islands. Aberrantly methylated genes participated in the activation of inflammation, sodium and potassium ion transport, fibrosis and the reduction of lipid metabolism. Hypermethylation in the AF susceptible loci, paired-like homeodomain transcription factor 2 (chromosome 4q25) and coiled-coil domain containing 141 (chromosome $2 \mathrm{q} 31$ ), as well as hypomethylation in the calcium voltage-gated channel subunit $\alpha 1 C$ (chromosome 12p13) locus, were identified in all patients with AF. Of the 420 upregulated and 567 downregulated genes previously identified in patients with AF relative to those with normal SR (fold-change $>2.0$; $\mathrm{P} \leq 0.05), 12$ genes were hypomethylated and eight genes were hypermethylated in each group, respectively $(|\beta|>0.2$ : $\mathrm{P}<0.05)$. RT-qPCR analysis of four of these genes supported the modulatory effect of DNA methylation on gene expression. These results suggest that DNA methylation-mediated
\end{abstract}

Correspondence to: Professor Pixiong Su, Department of Cardiac Surgery, Beijing Chaoyang Hospital, Capital Medical University, Ministry of Education, 8 Gongtinan Road, Chaoyang, Beijing 100020, P.R. China

E-mail: supixiong1130@163.com

Key words: atrial fibrillation, DNA methylation, pathogenesis regulation of gene expression may serve an important role in AF pathogenesis, and several susceptible AF CpG loci were identified which may be involved in the initiation of AF.

\section{Introduction}

Atrial fibrillation (AF) is the most common sustained type of cardiac arrhythmia occurring in 1-2\% of the general population worldwide, and is a major cause of morbidity and mortality (1). $\mathrm{AF}$ is a common phenotype that may occur as a result of a number of diverse pathophysiological conditions. AF is characterized by abnormalities in structure and electrophysiology in the left atrial tissue, which may result in abnormal impulse formation or propagation (2). AF is a highly heterogeneous genetic disease, which consists of distinct subtypes characterized by a few specific chromosomal abnormalities or gene mutations (3). Although candidate gene and genome-wide association studies (GWAS) have identified a number of genetic variants of AF, and elucidated the heritability of AF, a large proportion of AF cases cannot be explained by genetic variants alone (4).

Epigenetic regulation is an important and heritable mechanism of gene expression modulation. Cytosine methylation is thought to be a major mechanism underlying the epigenetic regulation of target genes, and DNA methylation serves important and dynamic roles in several cellular processes including, development, differentiation and DNA-protein interactions (5). Accumulating evidence has revealed that external stimuli, including environmental and life-style factors, may influence the gene expression status by disrupting DNA methylation patterns (6-8). This 'external stimulus DNA methylation gene expression' mode may explain the development of numerous diseases, such as cancer and autoimmune diseases. Cardiovascular diseases, including cardiac hypertrophy and atherosclerosis, involve aberrant DNA methylation patterns (9-12). The risk factors for AF include smoking, alcohol consumption and level of exercise, and AF tends to be heritable (13), as well as the incidence of potentially reversible AF conditions and subtypes without DNA sequence variation, indicate the participation of epigenetics in the pathogenesis of AF. However, the specific DNA methylation modifications that contribute to AF are currently unknown.

The present study compared the DNA methylome of left atrium (LA) tissue samples derived from patients with permanent AF (pAF) with healthy normal sinus rhythm (SR) control 
subjects using high-density methylation microarrays at a whole genome $\mathrm{CpG}$ range. $\mathrm{AF}$-associated biological processes and signaling pathways were further analyzed based on the differentially methylated genes. Genome-wide DNA methylation and the mRNA profile were further analyzed to determine the interaction between DNA methylation and gene expression.

\section{Materials and methods}

Collection of LA samples. There were 5 males in the patient group (aged 51-64) and 2 males in the healthy group (aged 48-53). Therefore, AF samples were obtained from seven patients diagnosed with pAF and coronary artery disease that had undergone LA radiofrequency ablation and mini incision coronary artery bypass grafting surgery. AF samples $(\sim 10 \times 5 \times 2 \mathrm{~mm})$ were obtained from the LA posterior wall between the inferior and superior right pulmonary veins and adjacent to the atriotomy line (14). Normal SR samples were obtained from the same site from four healthy heart donors that were not suitable candidates for heart transplantation procedures. Samples were snap frozen in liquid nitrogen and stored at $-80^{\circ} \mathrm{C}$ until required for further processing. All LA samples were obtained from patients admitted to the Department of Cardiac Surgery at Chaoyang Hospital (Capital Medical University, Beijing, China) between January 2013 and December 2013. The present study was performed in accordance with the Declaration of Helsinki guidelines for human studies, and was approved by the Ethics Committee of Chaoyang Hospital. All participants provided written informed consent for the collection of samples.

Genomic DNA and RNA extraction, and bisulfite conversion of $D N A$. Genomic DNA was isolated using proteinase K/RNase A treatment and phenol/chloroform extraction (DNeasy Blood \& Tissue kit; Qiagen Inc., Valencia, CA, USA). Total RNA was isolated using TRI Reagent BD (Sigma-Aldrich; Merck KGaA, Darmstadt, Germany) and an RNeasy Mini kit (Qiagen Inc.) according to the manufacturer's protocol. Following determination of sample DNA concentration, DNA quality was determined using a NanoDrop ND-1000 Spectrophotometer (Thermo Fisher Scientific, Inc., Wilmington, DE, USA), and DNA yields were analyzed by agarose gel electrophoresis. Once the quality control criteria had been met, where A260/A280>1.8 and A260/A230>2.0, DNA specimens $(1 \mu \mathrm{g})$ were subjected to bisulfite modification using an EZ DNA Methylation kit according to the manufacturer's protocol (Zymo Research Corporation, Irvine, CA, USA).

Microarray hybridization and analysis. The Illumina HumanMethylation450K BeadChip array (485,764 methylation sites, covering $99 \%$ of RefSeq genes; Illumina, Inc., San Diego, CA, USA) was employed for the purposes of the current study. This array covers $\mathrm{CpG}$ sites residing in a number of gene regions, including the promoter region, 5'-untranslated region (UTR), first exon, gene body and 3'-UTR. The DNA methylation assay, including the amplification, labeling and hybridization steps, was performed as previously described (15). Briefly, three levels of positive hybridization controls $(5,1$ and $0.2 \mathrm{pM})$, which perfectly complemented the sequence on the array, were synthesized and hybridized.
The positive controls exhibited a high signal intensity with a low background. The negative control, which used the probe sequence as a template, exhibited low signal intensity. These results indicated that the labeling and stripping reactions were successful. Bisulfite conversion efficiency was detected using the Infinium I probes and Infinium II probes (Illumina, Inc.), which were supplied with the microarray (16), which confirmed that the bisulfite conversion reaction was successful. Methylation data analysis including algorithms establishing project, defining group, normalization and differential methylation analysis were performed using GenomeStudio software (version, 2011.1; Illumina, Inc.) (16). Methylation results were reported as $\beta$-values $(0-1)$, which represented the fractional DNA methylation level of every $\mathrm{CpG}$ site to the total locus signal intensity. A relative methylation status was subsequently obtained between pAF and SR samples, with higher or lower values corresponding to hypermethylation or hypomethylation relative to the SR samples, respectively. Microarray analysis results were submitted to the Gene Expression Omnibus database (accession number, GSE62727; https://www.ncbi.nlm .nih.gov/geo/query/acc.cgi?acc=GSE62727).

Biological function analyses for differentially methylated genes. Gene ontology (GO) and pathway analyses were performed using the GO consortium (version number CC-BY 4.0; http://www.geneontology.org/) (15,17), Kyoto Encyclopedia of Genes and Genomes (KEGG; version 80.1; http://www.genome.jp/kegg/) (18) and Biocarta (http://www .biocarta.com/) online tools. Differential expression patterns were tested for biological processes and pathways displaying AF-related methylation patterns, with potential roles in the initiation and progression of AF. Signaling networks were constructed using the pathways identified with the KEGG database, in line with the association between differentially methylated genes, using the constellation graphical clustering method (version, 3.0; ClusterTech, Ltd., Hong Kong, China) (19).

Integrated analysis of genome-wide mRNA and methylation profiles in $A F$. In order to determine the association between DNA methylation and gene expression in AF, the correlation between the methylation analysis results of the present study and previously identified genome-wide mRNA microarray results involving the same samples (20), were compared and established. More stringent criteria, with mRNA expression values having a fold-change of $>2.0$ and a P-value of $\leq 0.05$, were considered to be statistically significant. Bioconductor software (https://www.bioconductor.org/) was utilized to reduce the potential impact of very high or low microarray values.

Reverse transcription quantitative-polymerase chain reaction $(R T-q P C R)$ validation of $m R N A$ expression levels. The expression pattern of four selected genes were validated by RT-qPCR analysis. RT and subsequent qPCR quantitation using SYBR Green PCR Master mix (Applied Biosystems; Thermo Fisher Scientific, Inc., Waltham, MA, USA) were performed as described previously (21). Primers were designed using the National Center for Biotechnology Information 'Primer-BLAST' tool (https://www.ncbi.nlm.nih .gov/tools/primer-blast/). A list of the primer sequences used is presented in Table I. Target gene expression levels were 
normalized against GADPH expression and quantified using the $2^{-\Delta \Delta \mathrm{Cq}}$ method (22). The results are presented as the mean of three independent experiments.

Statistical analysis. The results are presented as the mean \pm standard deviation. False discovery rates (FDR) were calculated to correct the P-value, due to the multiple comparisons performed in the microarray and functional analyses (23). The threshold P-value, FDR and $\beta$-values $(|\beta|)$ for differentially methylated $\mathrm{CpG}$ sites were set to $0.05,0.01$ and 0.17 (15), and the threshold P- and FDR values for GO items and pathway categories were set to 0.05 and 0.01 , respectively. Numerical data were analyzed using SPSS for Windows (version, 12.0; SPSS, Inc., Chicago, IL, USA). Student's t-test, Fisher's exact test and one-way analysis of variance followed by the Duncan's new multiple range post hoc test were used to determine differences in age, DNA methylation level and gene expression, GO category and relative expression between pAF and SR sample groups.

\section{Results}

Genome-wide DNA methylation pattern in $p A F$. The genome-wide DNA methylome of pAF and SR groups were first analyzed. No significant difference in global DNA methylation was detected $(\mathrm{P}>0.05)$; however, the average level in pAF samples $(16.6 \pm 1.7 \%)$ was slightly lower compared with SR samples (18.6 $\pm 2.4 \%)$. In total, 412 hypermethylated and 450 hypomethylated $\mathrm{CpG}$ sites (214 hypermethylated and 240 hypomethylated genes) were identified in fibrillating LA samples $(\mathrm{P}<0.05, \mathrm{FDR}<0.05,|\beta|>0.17$; data not shown $) ; 70.4$ and $56.0 \%$ of which were located in the gene coding regions (Fig. 1A and B). Based on the previously reported dysregulated genes in pAF (6), DNA methylation remodeling may affect a relatively small fraction of human genes.

To investigate the distribution of these $\mathrm{CpG}$ sites, two classification methods were used, which were based on their location relative to $\mathrm{CpG}$ islands (CGIs) and genes. The first method involved the following classifications: Within CGI; within CGI shore (0-2 kb from the CGI edge); within CGI shelf (>2-4 kb from the CGI edge); outside CGI. The second method used the following classifications: Within TSS1500 [200-1,500 bp upstream of the transcriptional start site (TSS)]; within TSS200 (1-200 bp upstream of the TSS); within the first exon; within the gene body (from the first intron to the last exon); within intergenic regions; within the 3'-UTR; within the 5'-UTR.

In general, the majority of the hypermethylated and hypomethylated $\mathrm{CpG}$ sites targeted gene-body and intergenic regions outside of CGIs (Fig. 1C-F). Of the 412 hypermethylated $\mathrm{CpG}$ sites, almost half (47.1\%) were located outside of CGIs (Fig. 1C), 189 (45.9\%) sites were located in the gene body and $133(32.3 \%)$ sites were located in the intergenic regions (Fig. 1D). Similarly, of the 450 hypomethylated $\mathrm{CpG}$ sites, $58.0 \%$ were outside of the CGIs (Fig. 1E), and $178(39.6 \%)$ and $177(39.3 \%)$ sites were located in the gene body and intergenic regions (Fig. 1F), respectively. A total of 90 and 38 hypermethylated and hypomethylated sites were located in CGIs, corresponding to 32 and 25 differentially expressed genes, respectively. Unsupervised hierarchical cluster analysis of differentially methylated $\mathrm{CpG}$ sites in the promoter regions revealed a clear separation between $\mathrm{pAF}$ and $\mathrm{SR}$ samples (Fig. 1G).

Biological function analysis of differentially methylated genes. To investigate the inter-association of the predominant biological processes and molecular functions in the differentially methylated genes, and to identify novel pathways associated with AF, all 454 differentially methylated genes were analyzed using the GO, KEGG and Biocarta databases. The immune response and antigen processing and presentation were the most prominent hypomethylation-associated GO biological processes $\left(\mathrm{P}<0.05, \mathrm{FDR}<10^{-33}\right.$; Fig. 2; Table II). This was exemplified by the hypomethylation of anoxia-reoxygenation associated genes in cardiac myocytes including proteasome subunit $\beta 9$ (Table II) (24), and human major histocompatibility complex (MHC) family members G, C, DMB, DRB1 and MHC-related protein 1 (20). The large-scale DNA hypomethylation detected in pAF-associated genes were associated with biological processes including sodium and potassium ion transport, cell differentiation, apoptosis and response to oxidation (Table II). Signaling pathways, including integrin-mediated signaling (25) and the transforming growth factor (TGF)- $\beta$ pathway (26), which serve essential roles in LA fibrosis, were observed to be hypomethylated (Table II). Cell adhesion and development were demonstrated to be the most prominent biological processes in hypermethylation-associated biological processes (Table II). Other enriched biological processes included metabolic pathways, such as lipid transport and lipoprotein metabolism. This was evidenced by the observed hypermethylation of key genes involved in lipid metabolism, including apolipoprotein L6, oxysterol binding protein like 3 and apolipoprotein A-V (Table II). The results of the biological function analyses indicated that DNA methylation may have an impact on LA remodeling in AF.

DNA methylation pattern in GWAS identified AF susceptible loci. Previous GWAS have identified several loci associated with pAF. Among these loci, paired like homeodomain transcription factor 2 (PITX2) $(4,27)$, coiled-coil domain containing 141 (CCDC141) and calcium voltage-gated channel subunit $\alpha 1 C$ (CACNA1C) (28) were all demonstrated to be differentially methylated in fibrillating LA when compared with SR, implying these genes may serve roles in AF. In the PITX2 locus, hypermethylation was located in CGIs (chromosome 4, 111,761,263-111,762,193 bp) and shore regions (chromosome 4, 111,542,062-111,544,464 bp) (data not shown). In CACNA1C and CCDC141 loci, hypermethylated and hypomethylated regions were located outside of the gene body, respectively (Fig. 3). The pathological roles of these genes are currently unknown.

Integrated analysis of genome-wide DNA methylation and gene expression profiles. To investigate the association between DNA methylation and gene expression in pAF, the methylation results were integrated with differential gene expression patterns previously identified in the same LA tissue samples (20). A total of 420 upregulated and 567 downregulated genes in $\mathrm{pAF}$ samples relative to SR samples were 
Table I. Primers for reverse transcription-quantitative polymerase chain reaction analysis of genes identified by integrated analysis of permanent atrial fibrillation and sinus rhythm groups.

\begin{tabular}{lllll}
\hline Gene & $\begin{array}{c}\text { GenBank } \\
\text { accession }\end{array}$ & \multicolumn{1}{c}{$\begin{array}{c}\text { Forward primer } \\
\text { sequence }(5 '-3 ')\end{array}$} & $\begin{array}{c}\text { Reverse primer } \\
\text { sequence }(5 '-3 ')\end{array}$ & $\begin{array}{c}\text { Forward/ } \\
\text { reverse } \\
\mathrm{T}_{\mathrm{m}}\left({ }^{\circ} \mathrm{C}\right)\end{array}$ \\
\hline HOXA3 & NM_153631 & CAGCCCTCTTTGGTCTAACT & GGTAAGGTCCGTGTAGGTGG & $60.9 / 64$ \\
C3orf59 & NM_178496 & CAGCATTGTCCTATCAGAAA & AAGATACCACAGGGACAATA & $58 / 56.4$ \\
RCAN1 & NM_004414 & TGACTGCGAGATGGAGGAGG & GGAGAAGGGGTTGCTGAAGT & $67.7 / 65.3$ \\
RUNX1 & NM_001001890 & GCAATGATGAAAACTACTCG & GTGATTTTGATGGCTCTGTG & $57.9 / 60.4$ \\
\hline
\end{tabular}

HOXA3, homeobox A3; RUNX1, runt-related transcription factor 1; RCAN1, regulator of calcineurin 1; $\mathrm{T}_{\mathrm{m}}$, primer melting temperature.

A

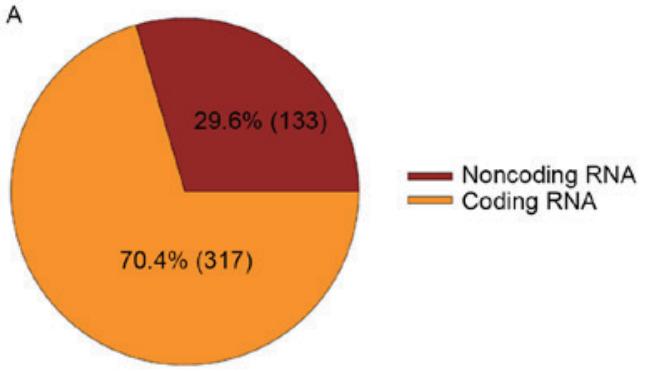

C

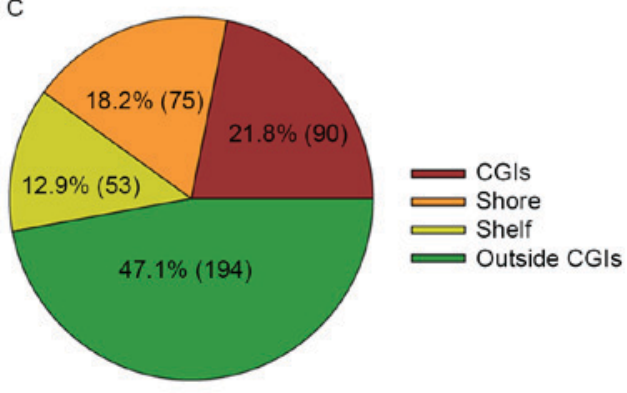

$\mathrm{E}$
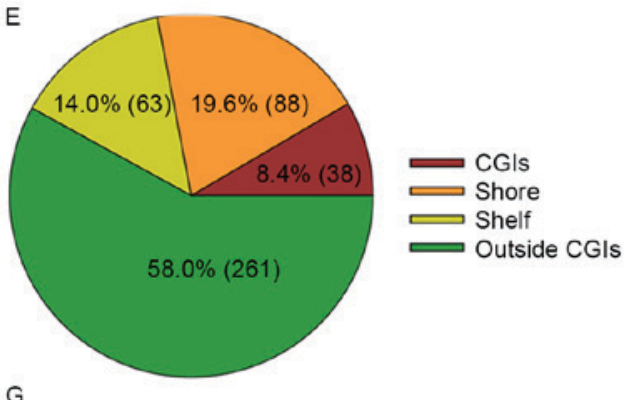

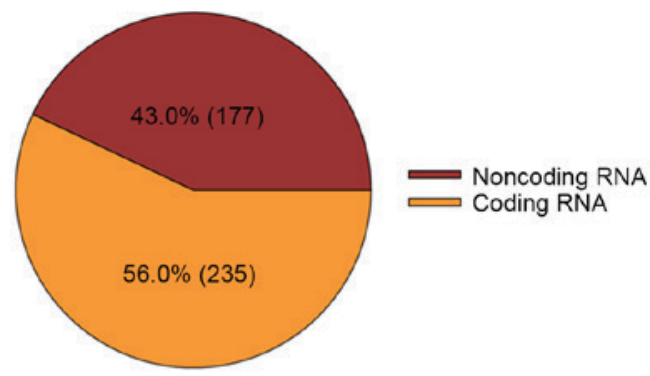

D
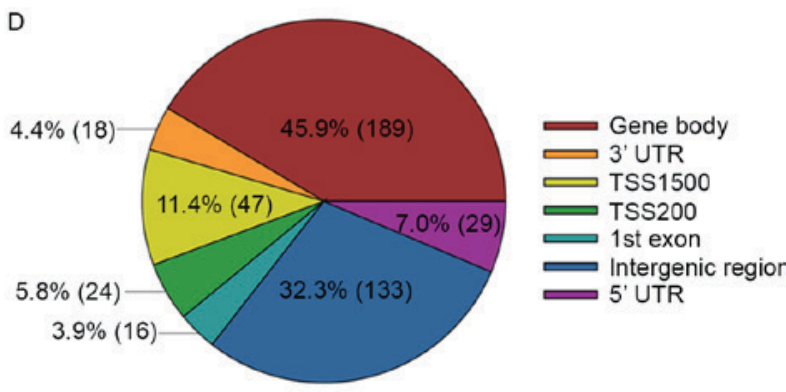

$\mathrm{F}$

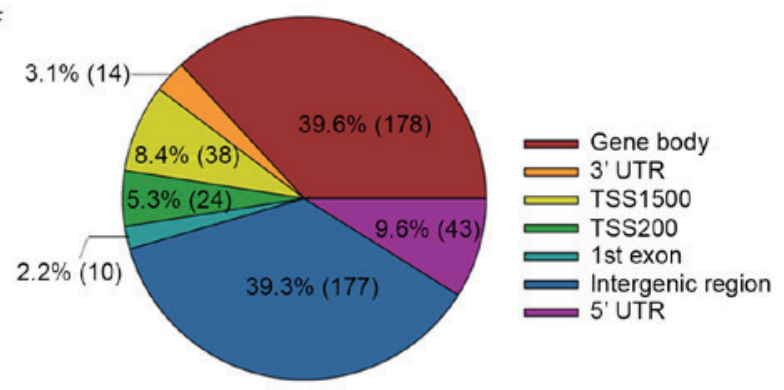

G
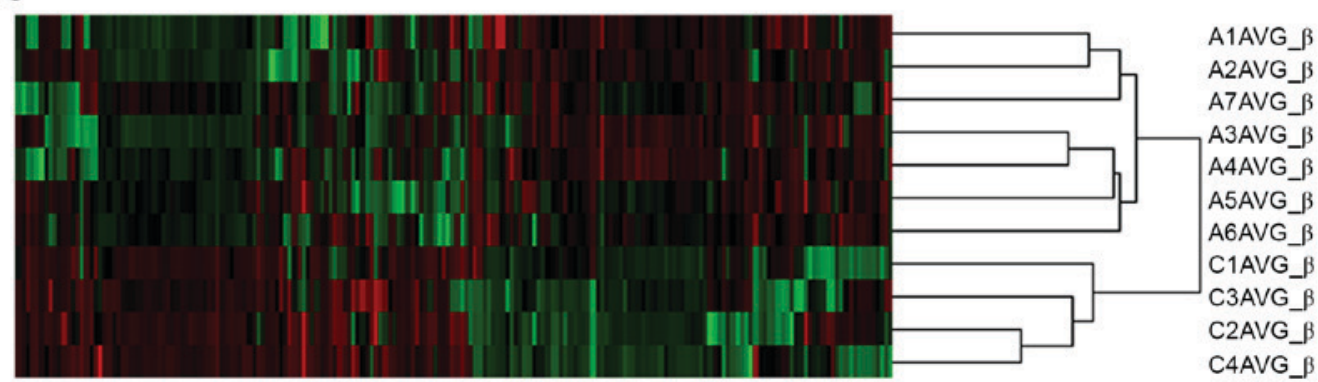

Figure 1. Genome-wide DNA methylation heterogeneity between pAF and SR samples. (A) Hypomethylated and (B) hypermethylated CpG sites of pAF compared with SR located in coding and noncoding RNA sequences. Location of hypermethylated CpG sites relative to (C) CpG islands and (D) genes. Location of hypomethylated $\mathrm{CpG}$ sites relative to $(\mathrm{E}) \mathrm{CpG}$ islands and (F) genes. Some CpG sites were assigned multiple annotations owing to the presence of alternative TSSs and regions containing $>1$ gene. $(\mathrm{G})$ Unsupervised hierarchical cluster analysis result of differentially methylated CpG sites in the promoter regions. pAF, permanent atrial fibrillation; SR, sinus rhythm; CGI, CpG island; UTR, untranslated region; TSS, transcriptional start site; TSS1500, 200-1,500 bp upstream of the transcriptional start site; TSS200, 1-200 bp upstream of the TSS. 
Table II. Biological function analysis of differentially methylated genes.

\begin{tabular}{|c|c|c|c|}
\hline Biological function & Methylation status & Count & Differentially methylated genes \\
\hline Immune response & Hypomethylated & 13 & $\begin{array}{l}\text { PSMB9, OPRD1, HLA-G, HLA-C, BMP6, } \\
\text { HLA-DMB, IL16, MR1, HLA-DRB1, } \\
\text { TAPBP, TGFBR3, HLA-DQA1, HLA-DRA }\end{array}$ \\
\hline Antigen processing and presentation & Hypomethylated & 5 & $\begin{array}{l}\text { HLA-DMB, HLA-DMB, HLA-DRB1, } \\
\text { HLA-DQA1, HLA-DRA }\end{array}$ \\
\hline Sodium ion transport & Hypomethylated & 4 & SLC12A7, SLC38A7, SLC9A9, SCN1A \\
\hline Potassium ion transport & Hypomethylated & 3 & KCNS3, KCNA4, ATP12A \\
\hline Cell differentiation & Hypomethylated & 8 & $\begin{array}{l}\text { GMCL1L, PPP1R9A, RNASE4, BMP8B, } \\
\text { PAX8, CYFIP1, HLX, MGP, }\end{array}$ \\
\hline Apoptosis & Hypomethylated & 6 & $\begin{array}{l}\text { STEAP3, CSRNP1, LGALS7, CLU, } \\
\text { DOCK1, UBE4B, }\end{array}$ \\
\hline Response to oxidative stress & Hypomethylated & 3 & MSRA, CLU, DUOX2 \\
\hline TGF- $\beta$ signaling pathway & Hypomethylated & 2 & BMP6, ВMP8B \\
\hline Integrin-mediated signaling pathway & Hypomethylated & 3 & ITGA5, ITGAE, DOCK1 \\
\hline Cell adhesion & Hypermethylated & 15 & $\begin{array}{l}\text { SIGLEC14, COL11A2, EFS, NLGN3, } \\
\text { ITGB4, AMIGO3, SIGLEC5, F3, } \\
\text { CTNNA2, NINJ2, DCHS2 }\end{array}$ \\
\hline Development & Hypermethylated & 10 & $\begin{array}{l}\text { SPDEF, HOXC4, NXN, PITX2, MSX1, } \\
\text { MEIS1, HDAC4, ARHGAP22, DUSP22, } \\
\text { HOXA3, EBF4, EBF3, PRM1, DMBX1 }\end{array}$ \\
\hline Lipid transport & Hypermethylated & 3 & APOL6, OSBPL3, APOA5 \\
\hline Lipoprotein metabolism & Hypermethylated & 2 & APOL6, APOA5 \\
\hline Notch signaling pathway & Hypermethylated & 2 & F3, MIB2 \\
\hline
\end{tabular}

Enrichment criteria threshold values included P-value of $<0.05$ and a false discovery rate of $<0.01$.

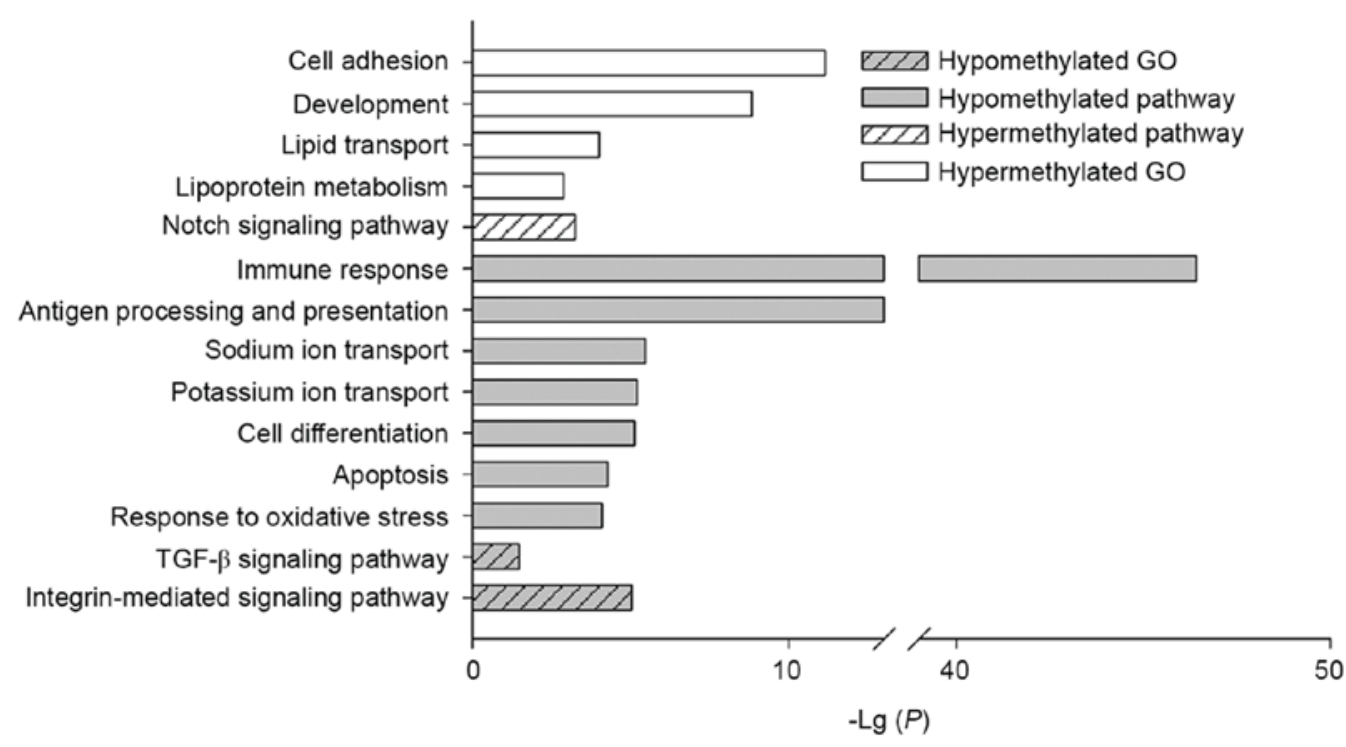

Figure 2. Analysis of the biological function of differentially methylated genes in permanent atrial fibrillation samples. The enrichment score represents the-log10 P-value, where P represents the significance testing value. TGF, transforming growth factor; GO, gene ontology.

identified $(\mathrm{P} \leq 0.05$, fold-change $>2.0)$. Of these genes, 12 and 8 were hypomethylated and hypermethylated, respectively $(\mathrm{P}<0.05, \mathrm{FDR}<0.05,|\beta|>0.2$; Table III $)$, and DNA methylation occurred in CGI and shore regions. The poor global association between DNA methylation and gene expression observed may have been due to the use of stringent criteria, or due to additional mechanisms of gene expression regulation. Of the 20 genes identified, runt-related transcription factor 1 (RUNX1) demonstrated the largest interaction with additional differentially methylated genes (Fig. 4). 

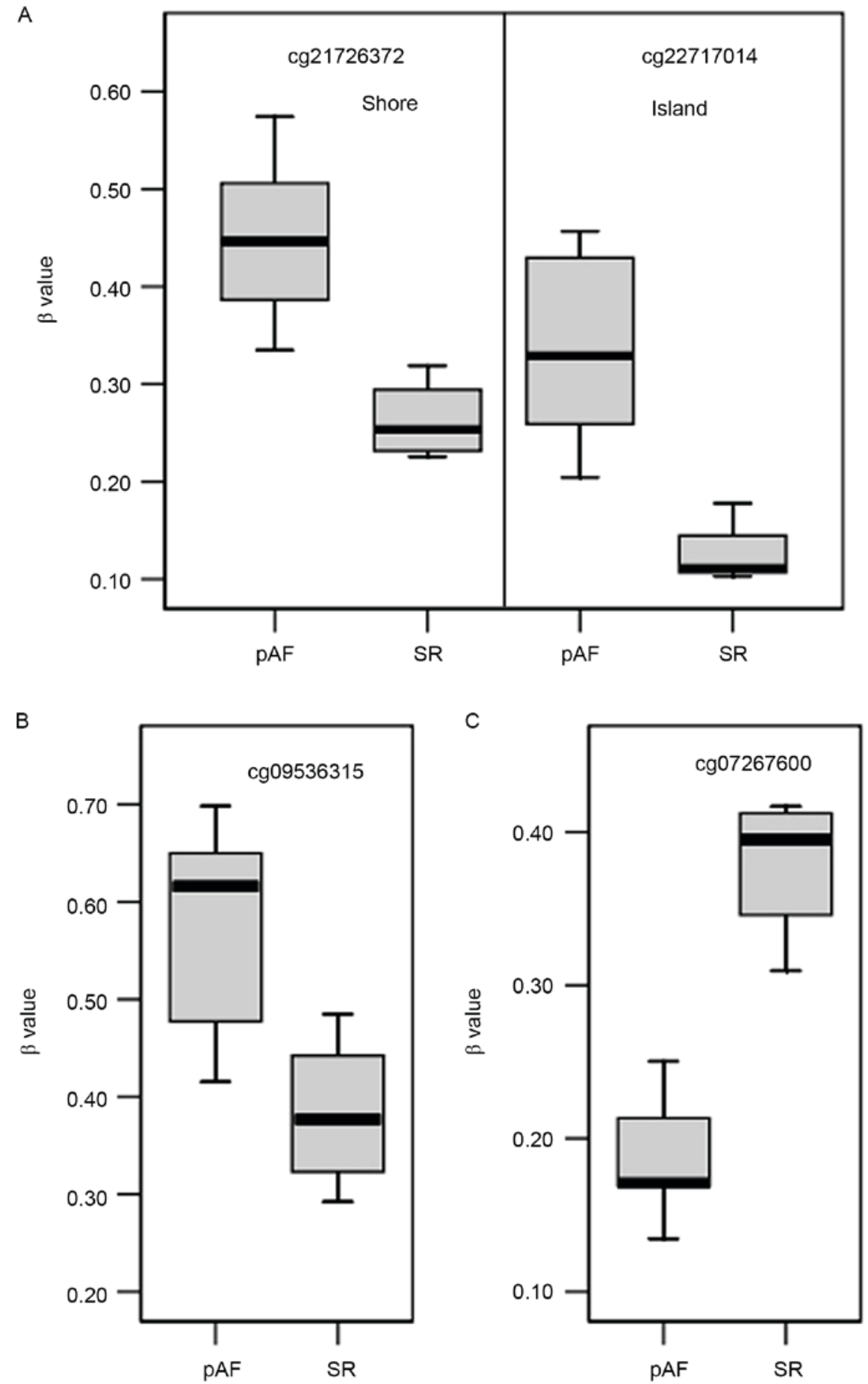

Figure 3. DNA methylation pattern in genome-wide association studies identified atrial fibrillation loci. DNA methylation levels are indicated on the y-axis. (A) Methylated $\mathrm{CpG}$ sites in paired like homeodomain transcription factor 2 loci (chromosome 4q25). (B) Methylated CpG sites in calcium voltage-gated channel subunit $\alpha 1 \mathrm{C}$ loci (chromosome 12p13). (C) Methylated $\mathrm{CpG}$ sites in coiled-coil domain containing 141 loci (chromosome 2q31). CGI, CpG island; pAF, permanent atrial fibrillation; SR, sinus rhythm. Data are presented as the mean \pm standard deviation.

$R T$-qPCR validation of AF-associated gene expression. To confirm the effects of DNA methylation on gene expression, the expression levels of one hypermethylated gene, homeobox A3, and three hypomethylated genes, C3orf59, RUNX1 and regulator of calcineurin 1 (Table III), were analyzed by RT-qPCR analysis. The results indicated that all four genes demonstrated an expression level that supported the modulatory effects of DNA methylation on gene expression in all pAF samples (Fig. 5).

\section{Discussion}

$\mathrm{AF}$ is a heterogeneous disease with a genetic component. Although some genetic studies have identified genes involved in the susceptibility and heritability of AF, only a small number of genes involved in the initiation and progression of $\mathrm{AF}$ have been identified thus far (29). DNA methylation is a heritable genome modification that does not involve nucleotide sequence alterations. Current studies have investigated the regulatory effects of 
Table III. Integrated analysis of genome-wide mRNA expression and methylation profiles in atrial fibrillation.

\begin{tabular}{|c|c|c|c|}
\hline Gene & GenBank accession & Methylation status & Location \\
\hline MAP4K1 & NM_001042600 & Hypermethylation & Body/island \\
\hline IL17RA & NM_014339 & Hypermethylation & 3'-UTR/shore \\
\hline MXRA7 & NM_001008529 & Hypermethylation & Body \\
\hline HOXA3 & NM_153631 & Hypermethylation & 5'-UTR/ island \\
\hline MXI1 & NM_130439 & Hypermethylation & Body/shelf \\
\hline ROCK2 & NM_004850 & Hypermethylation & Body \\
\hline CDKN1C & NM_000076 & Hypermethylation & TSS1500/island \\
\hline MAL2 & NM_052886 & Hypermethylation & TSS1500/shore \\
\hline PSMB9 & NM_002800 & Hypomethylation & Body/shore \\
\hline ABI3BP & NM_015429 & Hypomethylation & Body \\
\hline MOG & NM_206814 & Hypomethylation & Body \\
\hline POMZP3 & NM_152992 & Hypomethylation & Body/shelf \\
\hline SSTR4 & NM_001052 & Hypomethylation & TSS1500/shore \\
\hline LOC255167 & NR_024424 & Hypomethylation & TSS1500/shore \\
\hline C3orf59 & NM_178496 & Hypomethylation & Body \\
\hline JAKMIP3 & NM_001105521 & Hypomethylation & 3'-UTR \\
\hline BTNL9 & NM_152547 & Hypomethylation & 3'-UTR \\
\hline ZNF365 & NM_014951 & Hypomethylation & Body \\
\hline RCAN1 & NM_004414 & Hypomethylation & 5'-UTR/shelf \\
\hline RUNX1 & NM_001001890 & Hypomethylation & Body \\
\hline
\end{tabular}

Selection criteria for mRNA expression included a fold-change of $>2.0$ and a P-value of $\leq 0.05$. Selection criteria for the methylation profile included a P-value of $<0.05$, a false discovery rate of $<0.01$ and a $|\beta|$-value of $>0.2$. UTR, untranslated region; TSS1500, 200-1,500 bp upstream of the transcriptional start site.

DNA methylation in several mechanisms of disease. The present study performed genome-wide DNA methylation profiling, and analyzed the involvement of DNA methylation in pAF.

Despite analyzing a small number of samples, the results of the present study indicated that altered DNA methylation status may be a phenomenon in AF; however, no global patterns of hypomethylation or hypermethylation were identified. A total of 862 differentially methylated CpG sites between pAF and SR samples were detected, corresponding to 214 hypermethylated and 240 hypomethylated genes. Previous studies have reported $>1,000$ differentially expressed genes in pAF $(20,30)$. Therefore, DNA methylation alterations may directly impact upon a relatively small fraction of human genes in AF. The majority of the differentially methylated $\mathrm{CpG}$ sites were located in the gene body and intergenic regions outside of CGIs. DNA methylation in these regions is common in ubiquitous genes and facilitates transcription; however, a hypomethylated state in pAF may initiate transcription at incorrect sites (31). The results of the current study identified differential methylation sites located in CGIs. Over half $(\sim 60 \%)$ of gene promoters demonstrate a close association with CGIs, and unmethylated CGIs at promoters may promote transcription (32). A total of 32 out of 90 hypomethylated CGIs were located in promoter regions, corresponding to 26 genes, which included the fibroblast proliferation-associated gene galectin 8 and vimentin in previous studies $(33,34)$. A total of 25 hypermethylated CGIs were located in promoter regions, representing 18 genes, including integrin $\beta$ subunit 4 , which was previously demonstrated to exhibit reduced expression in pAF (35). Modulation of gene expression by DNA methylation in promoter regions may confirm previous observations of differentially expressed genes in LA. In the present study, the unsupervised hierarchical cluster analysis indicated that pAF and SR samples displayed distinct methylation patterns in promoter regions. In addition, aberrant methylation patterns were observed in shore and shelf regions.

The present study integrated genome-wide DNA methylation and gene expression analysis results, to assess the impact of methylation on gene expression regulation. Only 20 out of 454 differentially methylated genes were identified in both datasets. This poor association between DNA methylation and gene expression may have been due to stringent statistical index settings, or due to the participation of additional elements regulating gene expression. Among these 20 genes, the transcription factor, RUNX1, demonstrated the highest number of interactions with additional differentially methylated genes (Fig. 4). RUNX1 activates and promotes cardiac fibroblast proliferation via TGF- $\beta$ receptor II (36), signal transducer and activator of transcription 3 and the epidermal growth factor receptor; the latter two proteins are downstream signaling molecules of angiotensin II (37). In addition, RUNX1 inhibits cardioprotective effects from maladaptive hypertrophy via AKT serine-threonine kinase 3 (38) and transcription factor C/EBP (39). Furthermore, of the genes that were hypermethylated and downregulated, interleukin 17 receptor A induces interstitial myocardial fibrosis (40) and induces 


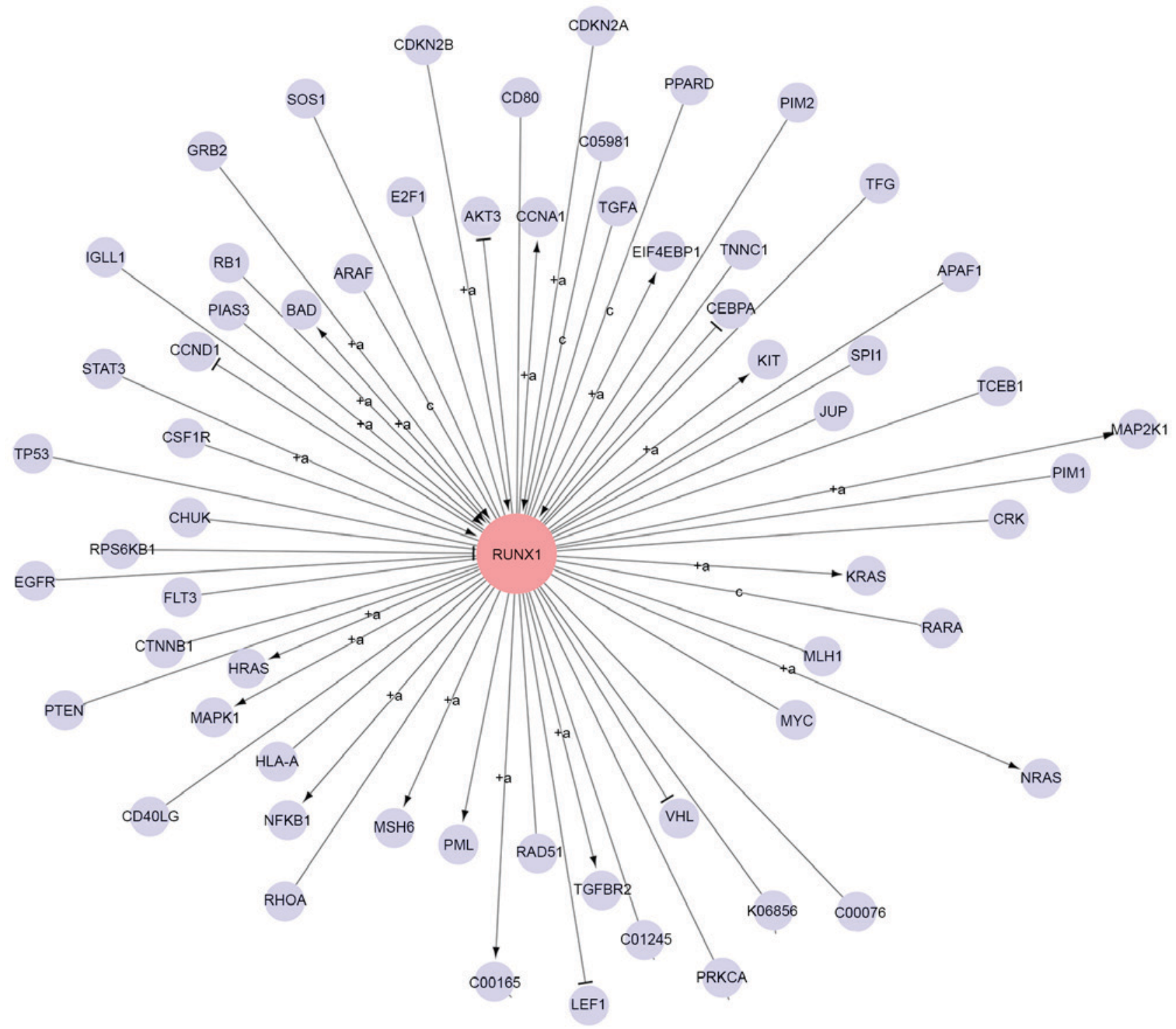

Figure 4. Signaling network of RUNX1 with additional differentially methylated genes. The circles represent genes and the lines represent interactions. Arrowheads indicate activation and rungs indicate inhibition. RUNX1, runt-related transcription factor 1; a, activation; c, compound.

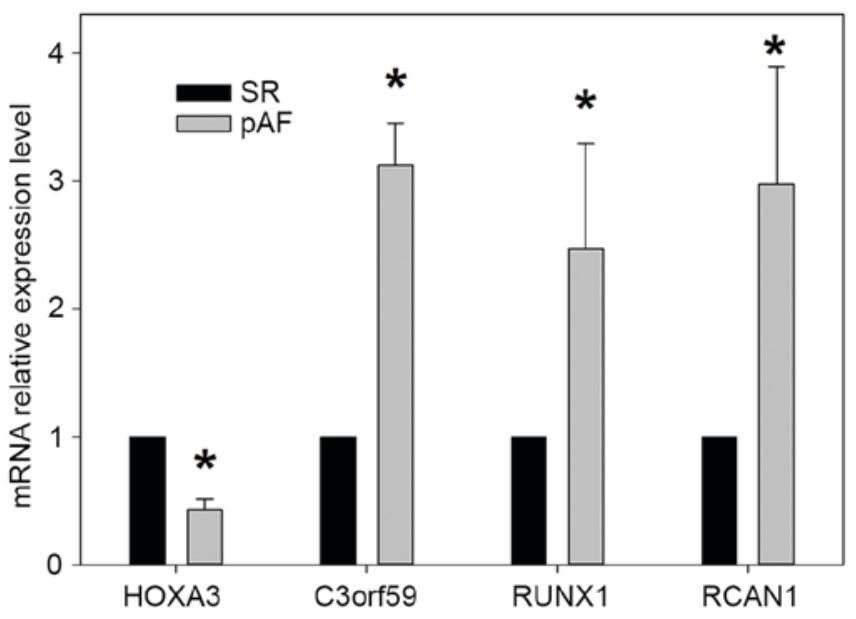

Figure 5. Relative expression of four selected genes (HOXA3, C3orf59, RUNX1 and RCAN1) identified by integrated analysis of pAF and SR groups. Values represent the mean + standard deviation. ${ }^{*} \mathrm{P}<0.05$ vs. SR group. HOXA3, homeobox A3; RUNX1, runt-related transcription factor 1; RCAN1, regulator of calcineurin 1; pAF, permanent atrial fibrillation; SR, sinus rhythm. inflammation via the production of chemokines and cytokines (41). Moreover, Rho-associated coiled-coil-containing protein kinase (ROCK) 2 mediates the contractile function and metabolism of the heart via the RhoA-ROCK signaling pathway (42). The methylation of susceptible loci in these genes may be an initiating event in AF upon exposure to an external stimulus.

However, the association between DNA methylation alterations and LA remodeling have not been clearly demonstrated thus far. The present study identified biological functions of differentially methylated CGIs, and revealed several potential key regulatory effects on the pathophysiology of AF.

The majority of the differentially methylated $\mathrm{CpG}$ sites in affected genes were associated with LA structural remodeling. Inflammation is considered to be the key factor in the initiation and perpetuation of AF (43); it confers a prothrombotic state that may lead to thrombotic complications. In the present study, the most prominent biological processes of hypomethylated $\mathrm{CpG}$ sites involved the immune response. It was reported that dysregulated DNA methylation of the IL-1 $\beta$ promoter and the 
proximal $\mathrm{CpG}$ motif-145C in the IL10 promoter may lead to an inflammatory state, where inflammatory cells are activated and release cytokines $(44,45)$. Potassium voltage-gated channel (KCN) S3, KCNA4 and sodium voltage-gated channel $\alpha$ (46) genes, which serve roles in sodium and potassium ion transport, were hypomethylated in the present study. This might induce conduction abnormalities and may explain the electrophysiological abnormalities of AF. In addition, processes including cell differentiation, apoptosis (47) and response to oxidation (48), which have previously been observed in LA tissues, were enriched biological processes among hypomethylated genes. Fibrosis is considered to be the hallmark of atrial remodeling, whereby the TGF- $\beta 1$ signaling pathway activates fibroblast proliferation and subsequent extracellular matrix production (26). Aside from angiopoietin II, activation of the TGF- $\beta 1$ pathway was previously reported to be mediated by the fibrotic cytokine, integrin- $\alpha 5$ (25); a key component of the integrin-mediated signaling pathway. In the present study, TGF- $\beta 1$ and genes involved in the integrin-mediated signaling pathways were hypomethylated. These differentially methylated CpG sites may present potential targets of anti-fibrosis therapies that are worthy of further investigation. Biological processes enriched across hypermethylated genes included lipid transport and lipoprotein metabolism, which are important processes in lipid metabolism (49). In the fibrillating atrium, the predominant energy source switches from lipid to glucose to compensate for the increased energy demand (50). Hypermethylation-mediated downregulation of lipid metabolism may be due to an initial compensatory mechanism to AF-induced hypermetabolic stress.

GWAS have revealed single-nucleotide polymorphisms located on chromosome $4 \mathrm{q} 25$, which are adjacent to PITX2 and were identified to be at a susceptible AF locus $(4,27)$. Although PITX2 gene knockout mice demonstrated increased susceptibility to atrial arrhythmias and arrhythmogenic alterations in action potentials $(51,52)$, PITX2 mutations were not identified in a cohort of AF patients (53). In the present study, pAF patients demonstrated significantly higher methylation levels in CGIs and shore regions of PITX2 when compared with SR participants. Therefore, DNA hypermethylation of the PITX2 locus may be an important phenomenon in AF, as opposed to gene mutation. However, the association between PITX2 and AF remains unknown. Hypomethylation and hypermethylation of the AF susceptible loci, CACNA1C and CCDC141, respectively were identified in the gene body which have demonstrated to be exert effects on cardiac conduction and rhythm disorders (28), and these differentially methylated genes may potentially serve roles in the arrhythmogenesis of AF.

In conclusion, the present study revealed that aberrant DNA methylation is a common phenomenon in the LA tissues of patients with AF. Although differential DNA methylation was only observed in a relatively small number of genes, DNA methylation-mediated regulation of gene expression may serve an important role in the pathogenesis of AF. In addition, the results identified methylation susceptible loci, which may be associated with the initiation and progression of AF, may be a therapeutic target in the treatment of pAF. However, whether DNA methylation regulation is one of the underlying factors contributing to $\mathrm{pAF}$ remains to be determined.

\section{Acknowledgements}

The current study was supported by grants from the National Science Foundation of China (grant no. 81070193), the Scientific Research Common Program of Beijing, Municipal Commission of Education (grant no. 3500-1142002801) and the Beijing Municipal Administration of Hospitals Clinical Medicine Development of Special Funding Support (grant no. XMLX201312, PS). The funders had no role in the study design, data collection and analysis, decision to publish, or preparation of the manuscript.

\section{References}

1. Manolis AJ, Rosei EA, Coca A, Cifkova R, Erdine SE, Kjeldsen S, Lip GY, Narkiewicz K, Parati G, Redon J, et al: Hypertension and atrial fibrillation: Diagnostic approach, prevention and treatment. Positionpaper of the working group 'hypertension arrhythmias and thrombosis' of the European society of hypertension. J Hypertens 30: 239-252, 2012.

2. January CT, Wann LS, Alpert JS, Calkins H, Cigarroa JE, Cleveland JC Jr, Conti JB, Ellinor PT, Ezekowitz MD, Field ME, et al: 2014 AHA/ACC/HRS guideline for the management of patients with atrial fibrillation: A report of the American college of cardiology/American heart association task force on practice guidelines and the heart rhythm society. J Am Coll Cardiol 64: e1-e76, 2014.

3. Fuster V, Rydén LE, Cannom DS, Crijns HJ, Curtis AB, Ellenbogen KA, Halperin JL, Kay GN, Le Huezey JY, Lowe JE, et al: 2011 ACCF/AHA/HRS focused updates incorporated into the ACC/AHA/ESC 2006 guidelines for the management of patients with atrial fibrillation: A report of the American college of cardiology foundation/American heart association task force on practice guidelines developed in partnership with the European society of cardiology and in collaboration with the european heart rhythm association and the heart rhythm society. J Am Coll Cardiol 57: e101-e198, 2011.

4. Magnani JW, Rienstra M, Lin H, Sinner MF, Lubitz SA, McManus DD, Dupuis J, Ellinor PT and Benjamin EJ: Atrial fibrillation: Current knowledge and future directions in epidemiology and genomics. Circulation 124: 1982-1993, 2011.

5. Messerschmidt DM, Knowles BB and Solter D: DNA methylation dynamics during epigenetic reprogramming in the germline and preimplantation embryos. Genes Dev 28: 812-828, 2014.

6. Benoit YD, Guezguez B, Boyd AL and Bhatia M: Molecular pathways: Epigenetic modulation of Wnt/glycogen synthase kinase-3 signaling to target human cancer stem cells. Clin Cancer Res 20: 5372-5378, 2014.

7. Jenkins TG, Aston KI, Pflueger C, Cairns BR and Carrell DT: Age-associated sperm DNA methylation alterations: Possible implications in offspring disease susceptibility. PLoS Genet 10: e1004458, 2014.

8. Rico-Rosillo G, Vega-Robledo GB, Silva-Garcia R and Oliva-Rico D: Epigenetics, environment and asthma. Rev Alerg Mex 61: 99-109, 2014 (In Spanish).

9. Mano H: Epigenetic abnormalities in cardiac hypertrophy and heart failure. Environ Health Prev Med 13: 25-29, 2008.

10. Papait R and Condorelli G: Epigenetics in heart failure. Ann N Y Acad Sci 1188: 159-164, 2010.

11. Yamada Y, Nishida T, Horibe H, Oguri M, Kato K and Sawabe M: MIdentification of hypo- and hypermethylated genes related to atherosclerosis by a genome-wide analysis of DNA methylation. Int J Mol Med 33: 1355-1363, 2014.

12. Nazarenko MS, Markov AV, Lebedev IN, Slepsov AA, Koroleva YA, Frolov AV, Barbarash OL, Barbarash LS and Puzirev VP: Methylation profile of INK4B-ARF-INK4A locus in atherosclerosis. Genetika 49: 783-787, 2013 (In Russian).

13. Palatini P: Parental atrial fibrillation as a risk factor for atrial fibrillation in offspring. JAMA 292: 1174-1175, 2004.

14. Corradi D, Callegari S, Maestri R, Benussi S, Bosio S, De Palma G, Alinovi R, Caglieri A, Goldoni M, Mozzoni P, et al: Heme oxygenase-1 expression in the left atrial myocardium of patients with chronic atrial fibrillation related to mitral valve disease: Its regional relationship with structural remodeling. Hum Pathol 39: 1162-1171, 2008. 
15. Ashburner M, Ball CA, Blake JA, Botstein D, Butler $\mathrm{H}$, Cherry JM, Davis AP, Dolinski K, Dwight SS, Eppig JT, et al: Gene ontology: Tool for the unification of biology. The gene ontology consortium. Nat Genet 25: 25-29, 2000.

16. Dedeurwaerder S, Defrance M, Calonne E, Denis H, Sotiriou C and Fuks F: Evaluation of the Infinium Methylation 450K technology. Epigenomics 3: 771-784, 2011.

17. Gene Ontology Consortium: Gene ontology consortium: Going forward. Nucleic Acids Res 43 (Database issue): D1049-D1056, 2015.

18. Kanehisa M, Furumichi M, Tanabe M, Sato Y and Morishima K KEGG: New perspectives on genomes, pathways, diseases and drugs. Nucleic Acids Res 45: D353-D361, 2017.

19. Zhu X, Wang H, Liu F, Chen L, Luo W, Su P, Li W, Yu L, Yang X and Cai J: Identification of micro-RNA networks in end-stage heart failure because of dilated cardiomyopathy. J Cell Mol Med 17: 1173-1787, 2013.

20. Zhou J, Gao J, Liu Y, Gu S, Zhang X, An X, Yan J, Xin Y and Su P: Human atrium transcript analysis of permanent atrial fibrillation. Int Heart J 55: 71-77, 2014.

21. Kaab S, Barth AS, Margerie D, Dugas M, Gebauer M, Zwermann L, Merk S, Pfeufer A, Steinmeyer K, Bleich M, et al: Global gene expression in human myocardium-oligonucleotide microarray analysis of regional diversity and transcriptiona regulation in heart failure. J Mol Med Berl) 82: 308-316, 2004.

22. Livak KJ and Schmittgen TD: Analysis of relative gene expression data using real-time quantitative PCR and the 2(-Delta Delta C(T)) method. Methods 25: 402-408, 2001

23. Hochberg Y and Benjamini Y: More powerful procedures for multiple significance testing. Stat Med 9: 811-818, 1990.

24. Kyrychenko VO, Nahibin VS, Tumanovs'ka LV, Dosenko VIe, Rybal'chenko VK and Moibenko OO: Effects of ubiquitin gene silencing in anoxia-reoxygenation of cultured cardiomyocytes. Fiziol Zh 56: 37-46, 2010 (In Ukrainian).

25. Zhao F, Zhang S, Shao Y, Wu Y, Qin J, Chen Y, Chen L, Gu H, Wang $\mathrm{X}$, Huang $\mathrm{C}$ and Zhang W: Calreticulin overexpression correlates with integrin- $\alpha 5$ and transforming growth factor- $\beta 1$ expression in the atria of patients with rheumatic valvular disease and atrial fibrillation. Int J Cardiol 168: 2177-2185, 2013.

26. Gu J, Liu X, Wang QX, Tan HW, Guo M, Jiang WF and Zhou L: Angiotensin II increases CTGF expression via MAPKs/TGF- $\beta 1 /$ TRAF6 pathway in atrial fibroblasts. Exp Cell Res 318: 2105-2115, 2012

27. Gudbjartsson DF, Arnar DO, Helgadottir A, Gretarsdottir S, Holm H, Sigurdsson A, Jonasdottir A, Baker A, Thorleifsson G, Kristjansson $\mathrm{K}$, et al: Variants conferring risk of atrial fibrillation on chromosome 4q25. Nature 448: 353-357, 2007.

28. den Hoed M,Eijgelsheim M,Esko T, Brundel BJ, PealDS, Evans DM Nolte IM, Segrè AV, Holm H, Handsaker RE, et al: Identification of heart rate-associated loci and their effects on cardiac conduction and rhythm disorders. Nat Genet 45: 621-631, 2013.

29. Husser D, Ueberham L, Dinov B, Kosiuk J, Kornej J, Hindricks G Shoemaker MB, Roden DM, Bollmann A and Büttner P: Genomic contributors to atrial electroanatomical remodeling and atrial fibrillation progression: Pathway enrichment analysis of GWAS data. Sci Rep 6: 36630, 2016.

30. Barth AS, Merk S, Arnoldi E, Zwermann L, Kloos P, Gebauer M, Steinmeyer K, Bleich $\mathrm{M}$, Kääb S, Hinterseer M, et al: Reprogramming of the human atrial transcriptome in permanent atrial fibrillation: Expression of a ventricular-like genomic signature. Circ Res 96: 1022-1029, 2005

31. Hellman A and Chess A: Gene body-specific methylation on the active X chromosome. Science 315: 1141-1143, 2007.

32. Illingworth RS, Gruenewald-Schneider U, Webb S, Kerr AR, James KD, Turner DJ, Smith C, Harrison DJ, Andrews R and Bird AP Orphan $\mathrm{CpG}$ islands identify numerous conserved promoters in the mammalian genome. PLoS Genet 6: e1001134, 2010

33. Ho JE, Yin X, Levy D, Vasan RS, Magnani JW, Ellinor PT, McManus DD, Lubitz SA, Larson MG and Benjamin EJ: Galectin 3 and incident atrial fibrillation in the community. Am Heart J 167: 729-734, 2014

34. Bunch TJ, Mahapatra S, Bruce GK, Johnson SB, Miller DV, Horne BD, Wang XL, Lee HC, Caplice NM and Packer DL: Impact of transforming growth factor-betal on atrioventricular node conduction modification by injected autologous fibroblasts in the canine heart. Circulation 113: 2485-2494, 2006.

35. Zhu H, Zhang W, Zhong M, Zhang G and Zhang Y: Differential gene expression during atrial structural remodeling in human left and right atrial appendages in atrial fibrillation. Acta Biochim Biophys Sin (Shanghai) 43: 535-541, 2011.
36. Tsai CT, Lai LP, Kuo KT, Hwang JJ, Hsieh CS, Hsu KL, Tseng CD, Tseng YZ, Chiang FT and Lin JL: Angiotensin II activates signal transducer and activators of transcription 3 via Racl in atrial myocytes and fibroblasts: Implication for the therapeutic effect of statin in atrial structural remodeling. Circulation 117: 344-355, 2008

37. Kim J, Eckhart AD, Eguchi S and Koch WJ: Beta-adrenergic receptor-mediated DNA synthesis in cardiac fibroblasts is dependent on transactivation of the epidermal growth factor receptor and subsequent activation of extracellular signal-regulated kinases. J Biol Chem 277: 32116-32123, 2002.

38. Taniyama Y, Ito M, Sato K, Kuester C, Veit K, Tremp G, Liao R, Colucci WS, Ivashchenko Y, Walsh K and Shiojima I: Akt3 overexpression in the heart results in progression from adaptive to maladaptive hypertrophy. J Mol Cell Cardiol 38: 375-385, 2005.

39. Huang GN, Thatcher JE, McAnally J, Kong Y, Qi X, Tan W, DiMaio JM, Amatruda JF, Gerard RD, Hill JA, et al: C/EBP transcription factors mediate epicardial activation during heart development and injury. Science 338: 1599-1603, 2012.

40. Baldeviano GC, Barin JG, Talor MV, Srinivasan S, Bedja D, Zheng D, Gabrielson K, Iwakura Y, Rose NR and Cihakova D: Interleukin-17A is dispensable for myocarditis but essential for the progression to dilated cardiomyopathy. Circ Res 106 1646-1655, 2010

41. Wu L, Ong S, Talor MV, Barin JG, Baldeviano GC, Kass DA, Bedja D, Zhang H, Sheikh A, Margolick JB, et al: Cardiac fibroblasts mediate IL-17A-driven inflammatory dilated cardiomyopathy. J Exp Med 211: 1449-1464, 2014.

42. Lin G, Brownsey RW and Macleod KM: Complex regulation of PKC 32 and PDK-1/AKT by ROCK 2 in diabetic heart. PLoS One 9: e86520, 2014

43. Issac TT, Dokainish $\mathrm{H}$ and Lakkis NM: Role of inflammation in initiation and perpetuation of atrial fibrillation: A systematic review of the published data. J Am Coll Cardiol 20: 2021-2028, 2007.

44. Fu LH, Ma CL, Cong B, Li SJ, Chen HY and Zhang JG: Hypomethylation of proximal $\mathrm{CpG}$ motif of interleukin-10 promoter regulates its expression in human rheumatoid arthritis. Acta Pharmacol Sin 32: 1373-1380, 2011.

45. Wessels I, Fleischer D, Rink L and Uciechowski P: Changes in chromatin structure and methylation of the human interleukin-1beta gene during monopoiesis. Immunology 130: 410-417, 2010.

46. Yamashita T, Murakawa Y, Hayami N, Fukui E, Kasaoka Y, Inoue $\mathrm{M}$ and Omata $\mathrm{M}$ : Short-term effects of rapid pacing on mRNA level of voltage-dependent $\mathrm{K}(+)$ channels in rat atrium: Electrical remodeling in paroxysmal atrial tachycardia. Circulation 101: 2007-2014, 2000.

47. Dispersyn GD, Ausma J, Thoné F, Flameng W, Vanoverschelde JL, Allessie MA, Ramaekers FC and Borgers M: Cardiomyocyte remodelling during myocardial hibernation and atrial fibrillation: Prelude to apoptosis. Cardiovasc Res 43: 947-957, 1999.

48. Rodrigo R: Prevention of postoperative atrial fibrillation: Novel and safe strategy based on the modulation of the antioxidant system. Front Physiol 3: 93, 2012.

49. Zhang S, Shen L, Xia Y, Yang Q, Li X, Tang G, Jiang Y, Wang J, Li M and Zhu L: DNA methylation landscape of fat deposits and fatty acid composition in obese and lean pigs. Sci Rep 6: 35063 , 2016.

50. Smetnev AS, Bunin IuA, Nargizian AB, Petrovskii PF and Vakhliaev VD: Characteristics of lactate metabolism in the myocardium of patients with auricular fibrillation). Kardiologiia 23: 70-73, 1983 (In Russian)

51. Kirchhof P, Kahr PC, Kaese S, Piccini I, Vokshi I, Scheld HH, Rotering H, Fortmueller L, Laakmann S, Verheule S, et al: PITX2c is expressed in the adult left atrium, and reducing Pitx $2 \mathrm{c}$ expression promotes atrial fibrillation inducibility and complex changes in gene expression. Circ Cardiovasc Genet 4: 123-133, 2011.

52. Wang J, Klysik E, Sood S, Johnson RL, Wehrens XH and Martin JF: Pitx 2 prevents susceptibility to atrial arrhythmias by inhibiting left-sided pacemaker specification. Proc Natl Acad Sci USA 107: 9753-9758, 2010

53. Boldt LH, Posch MG, Perrot A, Polotzki M, Rolf S, Parwani AS, Huemer M, Wutzler A, Ozcelik C and Haverkamp W: Mutational analysis of the PITX2 and NKX2-5 genes in patients with idiopathic atrial fibrillation. Int J Cardiol 145: 316-317, 2010. 